\title{
Alcohol Use History Not Available
}

National Cancer Institute

\section{Source}

National Cancer Institute. Alcohol Use History Not Available. NCI Thesaurus. Code C156823.

An indication that the alcohol use history of an individual is unavailable. 